\title{
Antenna Radiation Efficiency of the Korean NDGPS Based on Radiation Power Measurements
}

\author{
Young-Wan Kim, Member, KIICE \\ Department of Radio Communication Engineering, Kunsan National University, Kunsan 573-701, Korea
}

\begin{abstract}
The differential global positioning system (DGPS) transmits a GPS enhancement signal using a top-loaded monopole antenna in the medium frequency range. The top-loaded antenna in the medium frequency band can attain a radiation efficiency on the order of $10 \%$. The antenna ground plane characteristics affect the antenna radiation efficiency. To improve the radiation efficiency, it is necessary to install the antenna on a ground plane with large enough physical dimensions and good conductivity. The antenna radiation efficiency is a primary factor in determining the DGPS service area. The service area of the DGPS using a medium frequency band is dominantly affected by the antenna radiation efficiency. To determine antenna radiation efficiencies accurately, the antenna radiation efficiencies of DGPS are deduced from the propagation power in this paper. Based on the deduced antenna radiation efficiencies, the service area for the Korean nationwide-DGPS is analyzed and evaluated.
\end{abstract}

Index Terms: Nationwide differential global positioning system (NDGPS), DGPS antenna, Radiation efficiency, DGPS service area

\section{INTRODUCTION}

A differential global positioning system (DGPS) transmits an enhancement GPS signal in the frequency range of $283.5 \mathrm{kHz}$ to $325 \mathrm{kHz}$. The eleven ocean-based DGPS and the six land-based DGPS are in operation and providing differential GPS service in Korea [1]. The Korean DGPS service that covers the Korean peninsula has been operating since 2009. The ocean service area of ocean-based DGPS covers $100 \mathrm{NM}$, but the land-based DGPS covers a land service area of $80 \mathrm{~km}$ that cannot be serviced from an ocean-based DGPS. The DGPS service area is dependent from the radiation power of the DGPS via transmitting medium frequency (MF) antenna. The radiation power is the product of the output power of the DGPS and antenna radiation efficiency, which is the product of the antenna gain and antenna efficiency.
The DGPS transmitting antenna usually is the top-loaded antenna type [2, 3]. The ground side of the antenna is a series of radials that all group into a connection at the base of the antenna. The radial ground is installed as the same length as the transmitting antenna. Radials are crucial in the design of a top loaded antenna as the electrical properties of the antenna require a reaction between the top loaded portion and the ground system [3]. The top-loaded antenna in the medium frequency band gets a radiation efficiency on the order of $10 \%$. The antenna ground plane characteristics affect the antenna radiation efficiency. To improve the radiation efficiency, it is important to design the antenna with a proper electrical length. Also, it is necessary to install the antenna on the ground plane with a large enough physical size and a good conductivity. It is difficult to install the MF antenna with the required physical length. To extend the electrical length, a top-loaded antenna with an extension coil may be used as an MF DGPS antenna, although the

Received 07 March 2012, Revised 28 March 2012, Accepted 09 April 2012

*Corresponding Author E-mail:ywkim@kunsan.ac.kr 
antenna efficiency is decreased [4].

To predict the DGPS service area, it is important to obtain a more accurate radiation efficiency for an MF DGPS antenna. In this paper, the radiation efficiencies for the Korean DGPS are deduced from the DGPS radiation power. It is difficult to theoretically analyze the MF antenna radiation efficiency depending on the ground plane. Therefore, the radiation efficiency of the DGPS is analyzed based on radiation power measurements. The Korean DGPS service area is predicted from the deduced DGPS radiation efficiencies.

\section{MF ANTENNA AND WAVE PROPAGATION}

The MF antenna is designed by considering the ground plane as an element of the antenna. The antenna efficiency of the MF antenna is lower than that of a very high frequency (VHF) antenna. Therefore, the radiation efficiency determines the performance of the MF antenna, which considers the antenna gain and antenna efficiency as follows:

$$
\eta^{\prime}=G \cdot \eta / L
$$

where, $G$ is the antenna gain, $L$ is the propagation loss at the distance of $1 \mathrm{~km}$, which cannot be considered to be an efficiency factor. $\eta$ stands for the antenna efficiency.

The antenna efficiency, $\eta$ is expressed as follows:

$$
\eta=\frac{P_{r}}{P_{i}}=\frac{P_{r}}{P_{i}+P_{l}}
$$

where, $P_{i}$ is an input power for the antenna, $P_{r}$ is a radiation power, and $P_{l}$ is the antenna system losses that include losses due to the ground plane, a conductor loss of the antenna, and an antenna base insulator loss due to high frequency. The majority of the antenna system losses originate from the antenna ground plane.

From (1), the radiation efficiency is dependent on the antenna gain and the antenna efficiency. To improve the antenna gain, the antenna is designed with a proper antenna length. Also, the antenna efficiency is dependent on the conductivity and physical size of the ground plane. To enhance the radiation efficiency, therefore, the MF antenna should be installed on the ground plane with large enough physical dimensions and a good conductivity. The ground connection of the antenna is a series of radials that all group into a connection at the base of the antenna. The radial ground for MF antenna is shown in Fig. 1.

Assuming that the ground plane is a perfect conductor in Fig. 1, the MF antenna efficiency of (2) can be defined as:

$$
\eta=\frac{\operatorname{Real}\left(Z_{o}\right)}{\operatorname{Real}\left(Z_{a}\right)}=\frac{\operatorname{Real}\left(Z_{o}\right)}{\operatorname{Real}\left(Z_{a}+\Delta Z\right)}=\frac{R_{o}}{R_{o}+R_{d}}
$$

$$
\begin{gathered}
Z_{a} \equiv R_{a}+j X_{a} \quad Z_{o} \equiv R_{o}+j X_{o} \\
\Delta Z \equiv Z_{a}-Z_{o} \equiv R_{d}+j X_{d}
\end{gathered}
$$

where, $Z_{a}$ defines the feeder point impedance of the antenna and $Z_{o}$ represents the impedance of the ground plane. Also, $R_{O}$ is the radiation impedance of the antenna and $R_{d}$ is the grounding resistance.
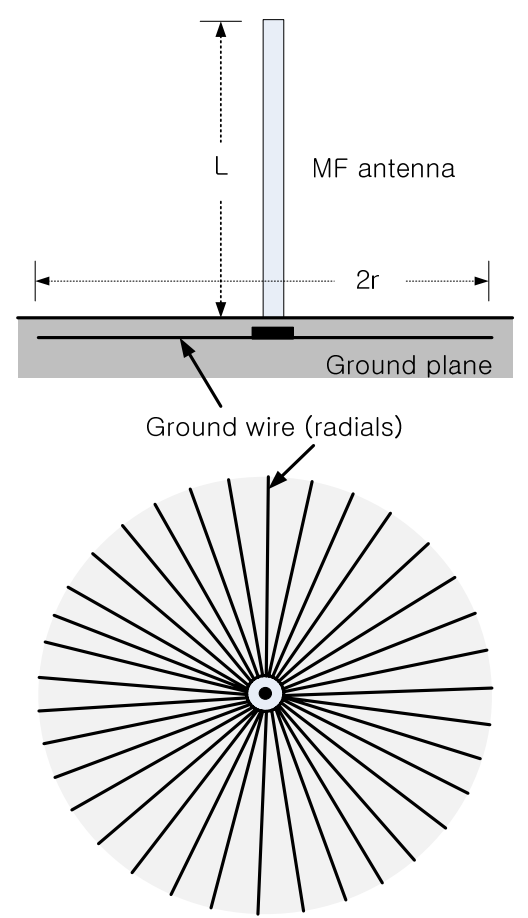

Fig. 1. Radial ground of the medium frequency (MF) antenna.

It is known from (3) that it is important to achieve a low grounding impedance, which is needed to have an adequate ground plane. In general, the radius (r) of the radial ground is designed over the length (L) of the MF antenna. The radials (ground wires) are installed 30 to $50 \mathrm{~cm}$ below the earth's surface. The number of the ground wires is used according to the output power capability of the DGPS, which is basically used with 60 to 240 wires.

In case the physical dimensions of the radial ground are small (in general $r<L$ ), the feeder point impedance of the antenna is seriously dependent on the status of the radial ground. Therefore, the radial ground without adequate physical dimensions has a serious influence on the antenna efficiency. The radiation efficiency of an MF antenna is increased as radials are installed on the ground plane with large enough physical dimensions. Provided that the MF antenna with a length near $0.6 \lambda$ is installed on the ground with a good conductivity, the MF antenna will have a high radiation efficiency (over $100 \%$ ) even on a small ground plane. The DGPS signal is propagated in free space via the MF antenna. Fig. 2 shows the ground wave configuration 
with a direct wave, a ground-reflected wave, and a surface wave [4].

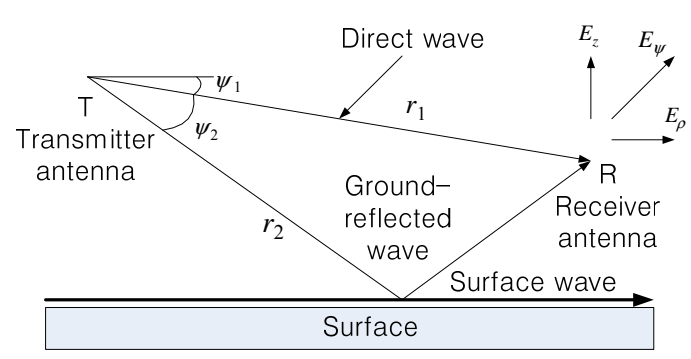

Fig. 2. Ground wave configuration.

From Fig. 2, the voltage $\mathrm{V}$ induced at the receiving antenna $\mathrm{R}$ from the transmitting antenna $\mathrm{T}$ with a distance of $r$ can be expressed as vector sum of a direct wave and a ground-reflected wave, and the surface wave $[5,6]$ :

$$
V=Q I\left\{Q_{1} \frac{\exp \left(-j k r_{1}\right)}{r_{1}}+Q_{2} \frac{\exp \left(-j k r_{2}\right)}{r_{2}}+S \frac{\exp \left(-j k r_{2}\right)}{r_{2}}\right\}
$$

where $I$ is the current at the transmitting antenna, $Q_{1}$ and $Q_{2}$ mean the polar diagram of the transmitting antenna and receiving antenna, respectively. $S$ denotes the surface wave component and takes various values according to the electrical characteristics of the ground surface, polarization, frequency, and the position of the transmitting antenna and receiving antenna.

In (4), the MF wave is propagated as surface wave using vertical polarization. The electric field intensity for the MF surface wave can be expressed as:

$$
\left|E_{z}\right|(m V / m)=\frac{300}{r} \sqrt{P}|A|
$$

where $P(\mathrm{~kW})$ is the radiation power and $r(\mathrm{~km})$ is the propagation distance of the MF wave. Also, A denotes the attenuation factor in the MF band.

The attenuation factor in the MF band is produced from the frequency $(f, \lambda)$, the propagation distance $(r)$, and the conductivity $(\sigma)$ and the dielectric constant $(\varepsilon)$ of the ground. The attenuation factor is expressed as $[5,6]$ :

$$
\begin{array}{ll}
A_{1(p, b)}=\frac{1}{2 p-3.7} & \left(b<5^{\circ}, p>45\right) \\
A_{2(p, b)}=e^{-0.43 p+0.01 p^{2}} & \left(b<5^{o}, \quad p \leq 45\right) \\
A_{3(p, b)}=e^{-0.43 p+0.01 p^{2}}-\frac{\sqrt{2}}{2} \sin b e^{\frac{-5 p}{8}} & \left(b>5^{o}, \quad p \leq 45\right) \\
A_{3(p, b)}=\frac{1}{2 p-3.7}-\frac{\sqrt{2}}{2} \sin b e^{\frac{-5 p}{8}} & \left(b>5^{o}, p>45\right)
\end{array}
$$

where the parameters in (6) are as follows:

$$
\begin{aligned}
& \chi=\frac{1.8 \times 10^{10} \cdot \sigma}{f} \\
& \tan b=\frac{\varepsilon+1}{\chi} \\
& p=\frac{\pi}{\chi} \cdot \frac{r}{\lambda} \cdot \cos b
\end{aligned}
$$

It is shown in form (6) that the attenuation factor is the function of the parameters of $b$ and $p$. It is clear from (7) that the parameters of $b$ and $p$ are dependent on the conductivity and the dielectric constant of the ground. To analyze the field intensity due to the propagation path, the proper conductivities and dielectric constants of propagation paths are considered in (7).

\section{MF FIELD INTENSITY AND RADIATION EFFICIENCY OF KOREAN DGPS}

To deduce the radiation efficiencies of the Korean DGPS, the electric field intensities for the Korean DGPS are measured at the proper points. Also, the electric field intensities for the measured points are theoretically produced from (5) and (6).

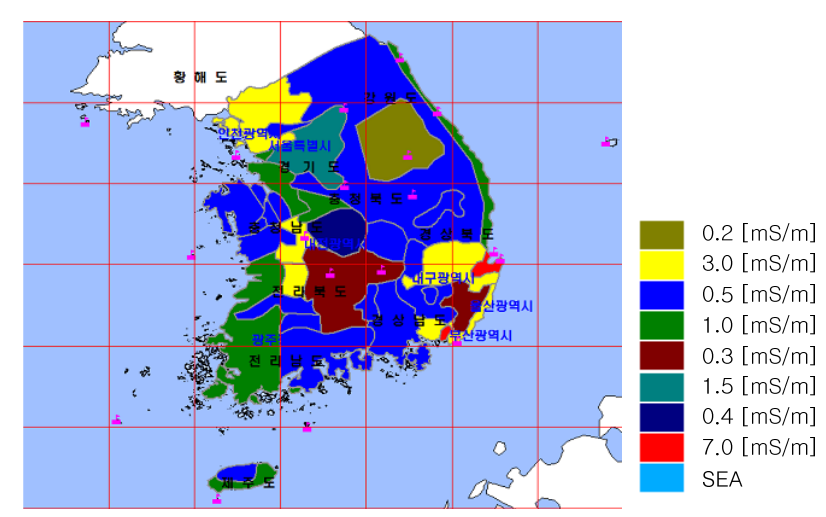

Fig. 3. Conductivity table of the Korean territory.

Then, the radiation efficiencies for the Korean DGPS of (1) are deduced by comparing the measured data and theoretical data. The conductivity data of the Korean territory is used in (7), which is shown in Fig. 3. From Fig. 3, Millington's method is adopted for the mixed paths, which means the propagation path including grounds with different conductivities [7].

Fig. 4 shows the measured data and theoretical data marked on the Korean map in the case of the Muju DGPS. The theoretical electric field intensities are produced for the same points (latitude and longitude) with measured points. To deduce the radiation efficiency of the DGPS, the data 
produced theoretically are compared on the map on which the measured data are marked.

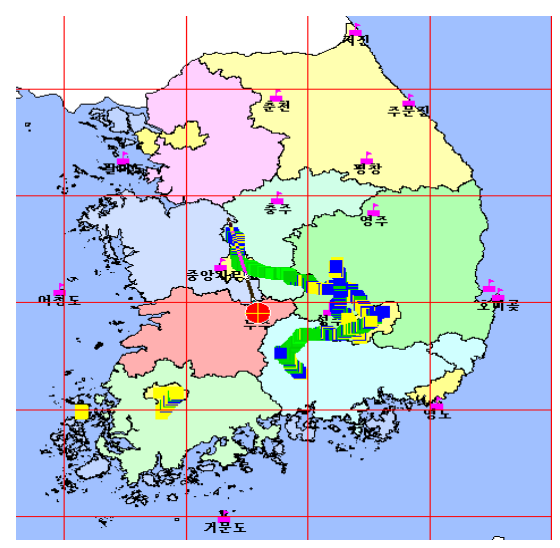

(a)

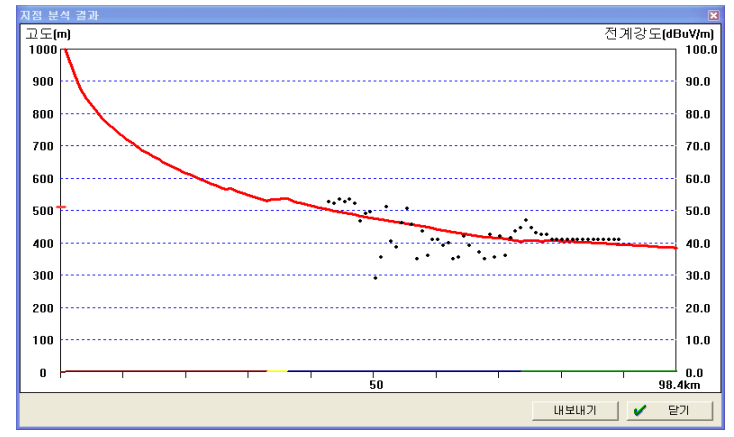

(b)

Fig. 4. Electric field intensity for Muju. (a) Data marked on a map of South Korea (b) data fitting of measured data and theoretical data.

The data fitting is accomplished over the propagation distance of $80 \mathrm{~km}$, which is the service area of a land-based DGPS. Fig. $4 \mathrm{~b}$ shows the theoretical data fitted to the measured data under the condition of a radiation efficiency of $40 \%$. It is shown from Fig. $4 \mathrm{~b}$ that the Muju DGPS with the radiation efficiency of $40 \%$ would radiate an MF wave that almost agrees with the measured electric field intensities.

From the data fitting of the measured data and theoretical data, the radiation efficiencies of the Korean DGPS can be deduced. Fig. 5 shows the data fitting for the land-based DGPS of Chungju and Chunchen. The radiation efficiencies of Chungju and Chunchen DGPS are $28.6 \%$ and $8.86 \%$, respectively. The antennas of the land-based DGPS are the top-loaded antenna type with the length of $90 \mathrm{~m}$, while the antenna of the Chunchen DGPS is new technology antenna (NTA) with a $24.9 \mathrm{~m}$ length. Therefore, it is clear that the radiation efficiency of the Chunchen DGPS is lower than that of the other land-based DGPS. This is because the antenna of the Chunchen DGPS is shorter in length.
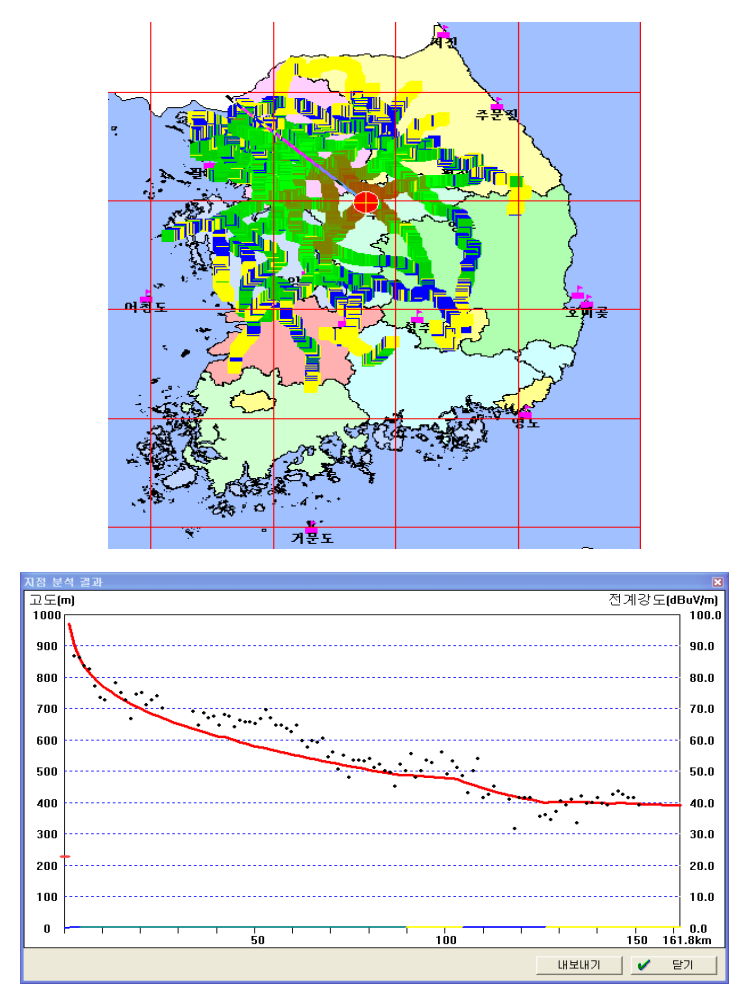

(a) Chungju
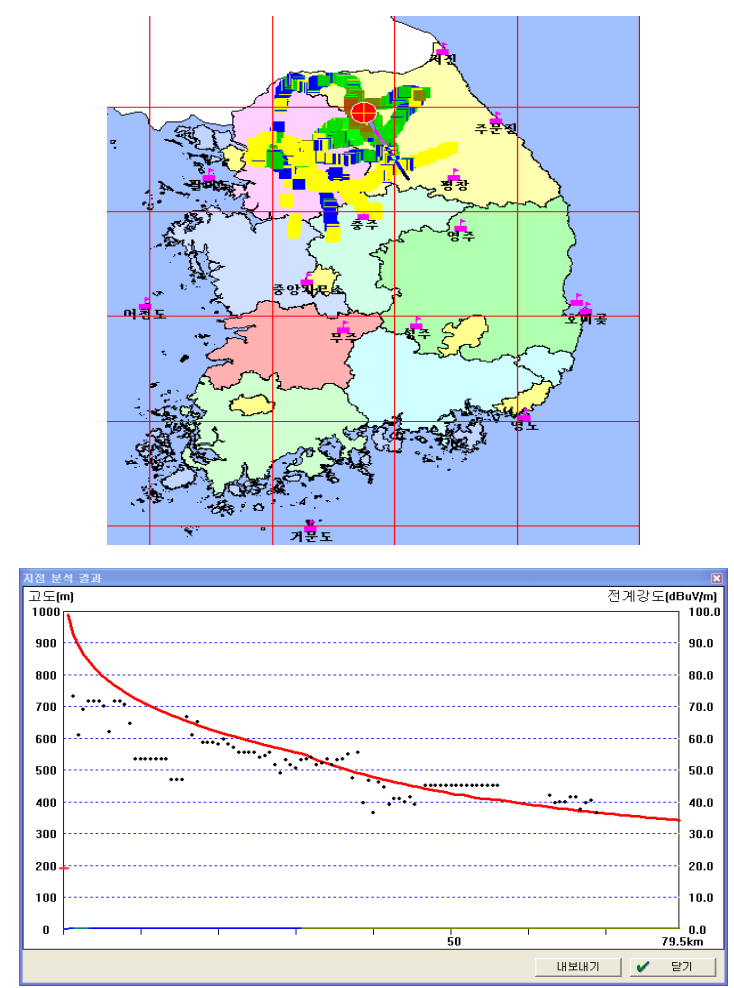

(b) Chunchen

Fig. 5. Data fitting of land-based differential global positioning system electric field intensities. 

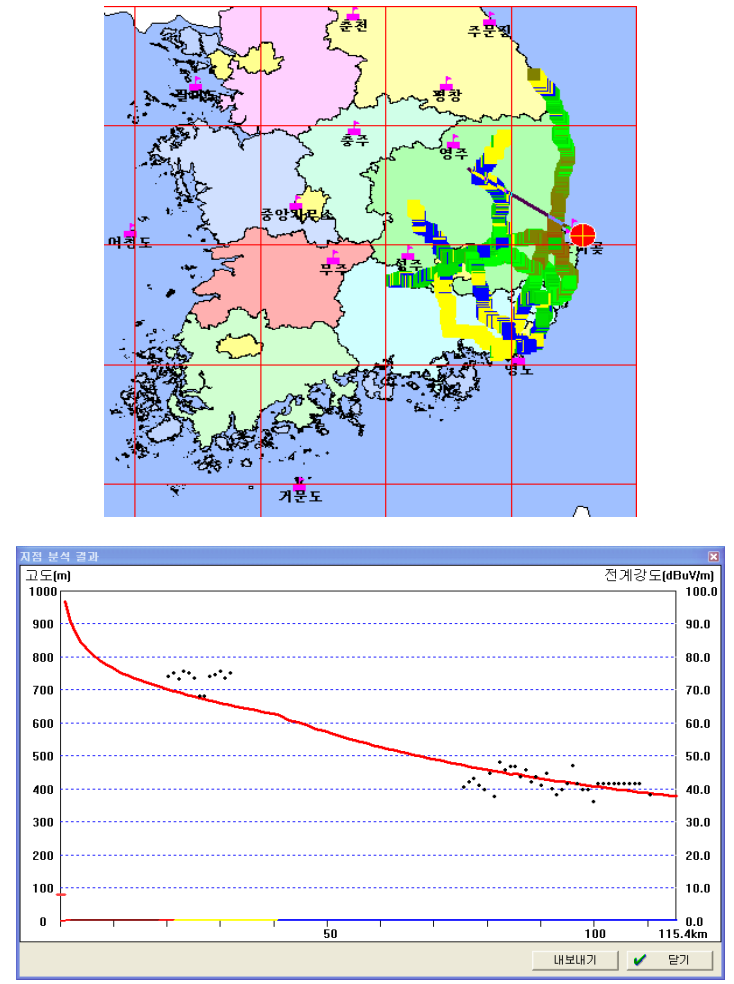

(a) Homigok
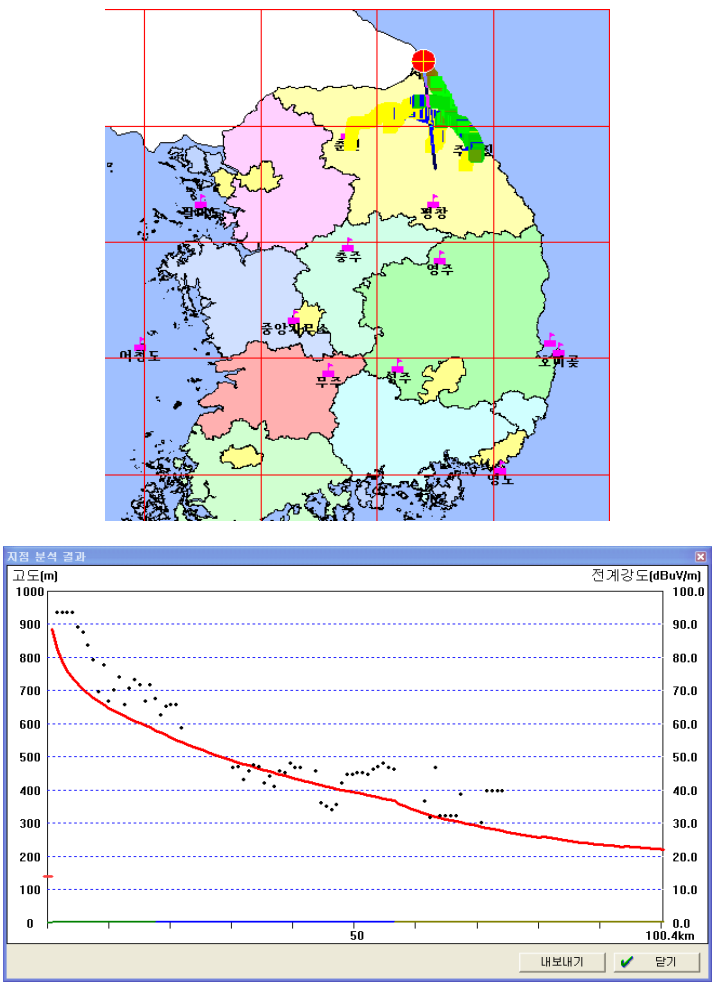

(b) Jeojin

Fig. 6. Data fitting of ocean-based differential global positioning system electric field intensities.
Fig. 6 shows the data fitting for the ocean-based DGPSs, which are installed at the seaside or on islands. It can be seen from Fig. 6 that the radiation efficiencies for oceanbased DGPSs are lower than those of land-based DGPS. In the ocean-based DGPSs, the shorter antennas are installed on the ground without large enough dimensions.

The radiation efficiencies of the ocean-based DGPS are in the range of 0.75 to $16 \%$.

By data fitting for all of the land-based DGPSs and ocean-based DGPSs, the radiation efficiencies for the Korean DGPS are deduced. Table 1 shows the radiation efficiencies for DGPS throughout the Korea.

Table 1. Radiation efficiencies for Korean differential global positioning system

\begin{tabular}{ccccc}
\hline Ref. station & Sochengdo & Palmido & Chungju & Echengdo \\
\hline $\begin{array}{c}\text { Radiation } \\
\text { efficiency } \\
\text { (\%) }\end{array}$ & 2 & 8 & 28.6 & 6.32 \\
\hline \hline Ref. station & Gemundo & Youngdo & Homigok & Ulleungdo \\
\hline $\begin{array}{c}\text { Radiation } \\
\text { efficiency } \\
(\%)\end{array}$ & 4.7 & 5 & 16 & 8 \\
\hline \hline Ref. station & Pyeongchang & Youngju & Muju & Sengju \\
\hline $\begin{array}{c}\text { Radiation } \\
\text { efficiency } \\
\text { (\%) }\end{array}$ & 14 & 22.1 & 40 & 28 \\
\hline \hline $\begin{array}{c}\text { Ref. station } \\
\text { Radiation } \\
\text { efficiency } \\
\text { (\%) }\end{array}$ & 3.63 & 0.75 & 1.89 & 2 \\
\hline \hline $\begin{array}{c}\text { Ref. station } \\
\text { Radiation } \\
\text { efficiency } \\
(\%)\end{array}$ & 8.86 & & & Jeojin \\
\hline
\end{tabular}

In Table 1, it can be seen that the Marado DGPS among the ocean-based DGPSs shows a very low radiation efficiency. The antenna of the Marado DGPS with a $24.9 \mathrm{~m}$ length was installed on the ground with a rock bed. Besides its shorter antenna length, the effect of a poor ground plane severely degrades the radiation efficiency of the Marado DGPS. Most of the ocean-based DGPS were installed on ground planes with low conductivities. It is clear that the DGPS MF antenna should be installed on the ground plane with large enough physical dimensions and good conductivity.

By applying the deduced radiation efficiencies, the service area of the Korean DGPS is predicted. Fig. 7 shows the service area of the Korean DGPS applying the deduced radiation efficiencies. In Fig. 7, there are some shadow areas in some parts of Kangwondo and Jeonnam. The factors affecting the shadow areas can be considered to be the propagation loss due to mountainous regions and radiation powers due to low radiation efficiencies. To enhance the 
service area, it is important to design the MF antenna system with a high radiation efficiency.

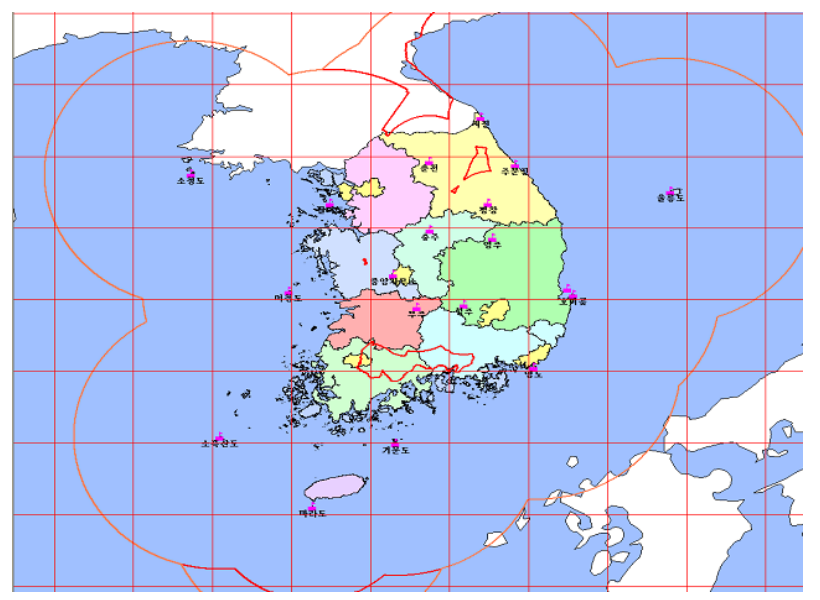

Fig. 7. Service area of Korean differential global positioning system applying deduced radiation efficiencies.

\section{CONCLUSIONS}

In this paper, the radiation efficiencies for Korean DGPS are deduced from the DGPS radiation power. The radiation efficiencies of the DGPS were analyzed based on the measured radiation power and theoretical electric field intensity.
The radiation efficiencies of the Korean ocean-based DGPSs were lower than those of the land-based DGPSs. In general, the ocean-based DGPSs were installed on narrow ground planes with low conductivities. It was concluded that to improve the radiation efficiency, an MF antenna should be designed with a proper antenna length and installed on a ground plane with good conductivity and large enough physical dimensions. Also, the Korean DGPS service area was predicted from the deduced DGPS radiation efficiencies. To enhance the service area, it is important to design the MF antenna system with a high radiation efficiency.

\section{REFERENCES}

[1] Differential Global Navigation Satellite System Central Office, Ministry of Land, Transport and Maritime Affairs [Internet]. Available: http://www.ndgps.go.kr/.

[2] V. Trainotti and L. A. Dorado, "Short low- and medium-frequency antenna performance," IEEE Antennas and Propagation Magazine, vol. 47 , no. 5 , pp. 66-90, 2005.

[3] V. Trainotti and L. A. Dorado, "On the crossed field antenna performance," IEEE Transactions on Broadcasting, vol. 52, no. 3, pp. 299-317, 2006.

[4] Y. W. Kim, "Propagation analysis of DGPS antenna for radial ground and obstacle," Journal of Information and Communication Engineering, vol. 9, no. 4, pp. 363-368, 2011.

[5] P. Knight and J. A. W. Robson, "Empirical formula for groundwave field-strength calculation," Electronics Letters, vol. 20, no. 18, pp. $740-742,1984$

[6] K. A. Norton, "The propagation of radio waves over the surface of the earth and in the upper atmosphere," Proceedings of the Institute of Radio Engineers, vol. 25, no. 9, pp. 1203-1236, 1937.

[7] D. Guerra, G. Prieto, I. Pena, S. Lopez, D. de la Vega, and P. Angueira, "Accuracy analysis of the Millington's prediction method in the medium waveband," IEEE Transactions on antennas and Propagation, vol. 55, no. 2, pp. 500-507, 2007.

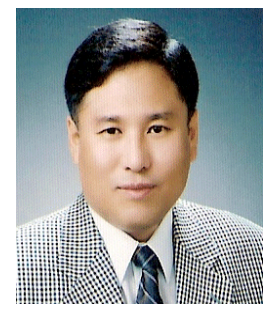

\section{Young Wan Kim}

received the M.S and Ph. D degrees in electronic engineering from Kyung-Pook National University, Taegu, and from Chung Nam National University, Daejeon, Korea in 1985 and 2003, respectively. From 1985 to 1992, he was with OPC Research Center, Korea, where he was involved in the development of the TVRO and the TVR system. From 1992 to 2004, he was with the Radio and Broadcasting Technology Lab., in the Electronics and Telecommunications Research Institute (ETRI) in Taejon, Korea. Since 2004, he has been with Kunsan National University and is presently a Professor in the Department of Radio Communication Engineering. His research interests are in digital satellite communication, TT\&C systems for satellites, digital broadcasting systems, radio communication technologies, and microwave devices. 